\title{
Adhesion of Membranes: A Theoretical Perspective
}

\author{
Reinhard Lipowsky ${ }^{*, \dagger}$ and Udo Seifert ${ }^{\ddagger}$ \\ Sektion Physik der Universität München, Theresienstrasse 37, \\ 8000 München 2, West Germany \\ Submitted to Symposium Chairman April 19, 1990. Received November 15, 1990. \\ In Final Form: February 22, 1991
}

\begin{abstract}
We theoretically study (i) a large membrane segment and (ii) a closed membrane surface or vesicle that adhere to another surface. The membrane segment can undergo unbinding transitions as a result of thermally excited shape fluctuations. These transitions are studied by renormalization group methods and by Monte Carlo simulations. The shape of a bound vesicle is determined by the interplay of bending and adhesion energies. This interplay leads to adhesion transitions from a free to a bound vesicle state even in the absence of shape fluctuations. Our theory helps to clarify the notion of a contact angle for membranes.
\end{abstract}

\section{Introduction}

Adhesion of membranes plays an essential role for many biological, biochemical, and biophysical phenomena. For example, the formation of tissue is based upon the mutual adhesion of cell membranes or on the adhesion of these membranes to a network of macromolecules. On a somewhat smaller scale, many transport processes involve the binding and unbinding of vesicles to and from the membrane surfaces of cells and organelles. This latter process can be used for the delivery of drugs to specific cell types. Another example is the construction of biosensors, which is often based on the binding of membranes to solid surfaces.

In this paper, we discuss recent theoretical work on the adhesion of membranes. First, we briefly review the elastic properties of fluid and polymerized (or tethered) membranes in section II. Then, we consider two relatively simple adhesion problems: (i) The adhesion of a large membrane segment to another surface is studied in section III. The shape of the membrane is not fixed but undergoes thermally excited shape fluctuations that act to renormalize the direct interaction between the surfaces. ${ }^{1}$ This renormalization can lead to unbinding transitions from bound to unbound states of the membrane. ${ }^{2,3}$ (ii) The adhesion of a closed membrane surface or vesicle to another surface is considered in section IV. The shape of such a vesicle is determined by the interplay of adhesion and bending energies. ${ }^{4}$ In contrast to the usual wisdom, we find that the notion of a contact angle is not valid in general. Furthermore, the vesicle may unbind from the wall even in the absence of shape fluctuations.

\section{Fluid and Polymerized Model Membranes}

The lateral extension of lipid bilayers is typically of the order of $1-10 \mu \mathrm{m}$ while their molecular thickness is $\simeq 4$ $\mathrm{nm}$. Therefore, such a membrane represents a nearly twodimensional sheet of molecules. In aqueous solution, such bilayers form closed surfaces or vesicles, the shape of which

\footnotetext{
1 New address: Institut für Festkörperforschung, Forschungszentrum Jülich, 5170 Jülich, Germany.

₹ Present address: Department of Physics, Simon Fraser University, Burnaby, BC, Canada V5A 1 S6.

(1) For a review, see Lipowsky, R. In Random Fluctuations and Growth; by Stanley, H. E., Ostrowsky, N., Eds.; Kluwer Academic Publishers: Dordrecht, 1988; Phys. Scr., T 1989, $29,259$.

(2) Lipowsky, R. Europhys. Lett. 1988, 7, 255; Phys. Rev. Lett. 1989 62,704
}

(3) Lipowsky, R.: Zielinska, B. Phys. Rev. Lett. 1989, 62, 1572.

(4) Seifert, U.; Lipowsky, R. Phys. Rev. A 1990, 42, 4768. has been studied in many experiments. ${ }^{5}$ These studies support the theoretical concept that the average shape of membranes is controlled by bending rigidity. ${ }^{6}$ Likewise, typical shape fluctuations of membranes represent thermally excited bending modes.

The bending modes of a single membrane can be described by a variable, $z(x)$, which measures the local distance of the membrane from a reference plane. The associated bending energy depends on the internal structure of the membrane. From a theoretical point of view, two model membranes can be distinguished: (i) fluid and (ii) polymerized or tethered membranes.

For a fluid membrane, the bending energy per unit area is given by $(\kappa / 2)\left(\nabla^{2} z\right)^{2}$ where $\kappa$ is the bending rigidity and $\nabla^{2} z$ is the leading term of the mean curvature. This form is valid for wavenumbers $q \gtrsim 1 / \xi_{\mathrm{p}}$ where the length scale $\xi_{\mathrm{p}} \sim a \exp [2 \pi \kappa / T]$ represents the persistence length of the membrane at temperature $T$ (which is measured in units of the Boltzmann constant $\left.k_{\mathrm{B}}\right) .^{7}$ On length scales large compared to $\xi_{\mathrm{p}}$, the membrane is expected to crumple and thus to lose its orientational order. ${ }^{8}$

For usual lipid bilayers in the fluid $\left(\mathrm{L}_{\alpha}\right)$ phase, the persistence length $\xi_{\mathrm{p}}$ is expected to be very large. For example, the (bare) bending rigidity of lecithin is believed to be $\kappa \simeq 10^{-19} \mathrm{~J}$, which implies $\kappa / T \simeq 20$ and $\xi_{\mathrm{p}} / a \simeq 10^{54}$ at room temperature. Even if $\alpha$ were only $\simeq 10^{-20} \mathrm{~J}$ as might be appropriate for DGDG membranes, ${ }^{9} \xi_{\mathrm{p}}$ /a would still be $\simeq 10^{5}$ and, thus, $\xi_{p}$ would still be larger than the size of large vesicles. Therefore, for lipid bilayers at room temperature, the accessible scales are usually much smaller than the persistence length. The same situation presumably applies to many biological membranes. ${ }^{10}$

Lipid bilayers often exhibit a crystalline $\left(\mathrm{L}_{\beta}\right)$ phase in which the molecules form a triangular lattice and cannot diffuse freely. Free diffusion is also suppressed in poly-

(5) See, e.g., Physics of Amphiphilic Layers; Meunier, J., Langevin, D., Boccara, N., Eds.; Springer Proceedings in Physics; Springer-Verlag: Berlin, 1987; Vol. 21.

(6) Helfrich, W. Z. Naturforsch., C: Biosci. 1973, 28c, 693.

(7) de Gennes, P.-G.; Taupin, C. J. Phys. Chem. 1982, 88, 2294.

(8) For fluid membranes, the effect of self-avoidance on crumpling is not well understood. For tethered membranes, self-avoidance seems to prevent any crumpling, see Abraham, F. F.; Rudge, W. E.; Plischke, M. Phys. Rev. Lett. 1989, 62, 1757. Ho, J. S.; Baumgärtner, A. Phys. Rev. Lett. 1989, 63, 1324.

(9) Mutz, M.; Helfrich, W. Phys. Rev. Lett. 1989, 62, 2881.

(10) An exceptionally small persistance length seems to apply to DMPC membranes with a small amount of dipolar ("bola") lipid which have been recently studied by H. P. Duwe, J. Kaes, and E. Sackmann, (to be submitted for publication). In this case, the bending stiffness seems to be of order $T$. In fact, the shape fluctuations are so strong that the vesicles become unstable and expel the bipolar lipid in the form of small vesicles. 
merized membranes in which the lipid molecules are crosslinked by chemical bonds. ${ }^{11}$ In this case, the strain tensor of the membrane contains a term that couples the membrane displacement, $z$, to the internal displacement fields. ${ }^{12}$ This leads to an effective scale-dependent rigidity, $\kappa_{\text {eff }}(q) \sim K / q^{\eta}$ for small wavenumbers, $q$, with $\eta>0 .{ }^{13 a}$ Computer simulations of tethered networks gave the estimate $\eta \simeq 0.74$, which would imply that the shear modulus vanishes on large scales. ${ }^{14}$ More recently, Monte Carlo simulations of solidlike elastic sheets gave $\eta=1$ for polymerized membranes, which is consistent with a finite shear modulus on large scales. ${ }^{15}$

For a flat crystalline membrane, a single dislocation has a stretching energy that diverges logarithmically with the membrane size. This energy is reduced if the membrane is allowed to buckle. In fact, recent calculations indicate that, for a buckled membrane, the energy of such a dislocation might be finite. ${ }^{13 b}$ Dislocations would then be thermally activated and the membrane would break up into crystalline domains with a characteristic size, $\xi_{\mathrm{c}}$. This length scale is set by the mean separation of the dislocations; for lipid bilayers at room temperature, it is expected to be $\xi_{c} \simeq 100 a$. On scales large compared to $\xi_{c}$, the membrane could represent a hexatic phase, which is characterized by a finite bending rigidity and thus by $\eta$ $=0.13 \mathrm{a}, \mathrm{c}$

\section{Renormalized Interaction and Unbinding Transition}

The direct interaction, $V_{\mathrm{DI}}(l)$, between two planar membranes can be determined experimentally when the membranes are immobilized on molecular smooth mica surfaces. The force between two such surfaces can then be measured quite accurately as a function of the surface separation. For example, rather precise data on the van der Waals attraction between two immobilized bilayers has been obtained in this way. ${ }^{16}$

In solution, bilayers do not stay planar, however, but undergo shape fluctuations. From a theoretical point of view, this represents a challenging problem since it involves many length scales. Indeed, the smallest wavelength of the membrane fluctuations is set by the size, $a \sim 1 \mathrm{~nm}$, of the amphiphilic molecules while their largest wavelength is of the order of the membrane dimension, which is typically $\sim 1-10 \mu \mathrm{m}$. Thus, the fluctuations are composed of many bending modes with wavenumber $1 / L \leqslant q \leqslant 1 / a$.

These fluctuations renormalize the direct interaction, $V_{\mathrm{DI}}$, in a nontrivial way. ${ }^{1-3,17}$ The renormalized interaction may be attractive or repulsive at large membrane separation corresponding to a bound or an unbound state of the membranes. These two different states are separated by a phase boundary at which the membranes undergo an unbinding transition. Such transitions were first predicted theoretically on the basis of renormalization group (RG) calculations both for fluid ${ }^{2,17}$ and for polymerized (or tethered or crystalline) ${ }^{2}$ membranes. For fluid membranes, the existence of these transitions has been confirmed by computer simulations ${ }^{3}$ and by experiments on

(11) See, e.g., Sackmann, E.; Eggl, P.; Fahn, C.; Bader, H.; Ringsdorf, H.; Schollmaier, M. Ber. Bunsen-Ges. Phys. Chem. 1985, 89, 1198.

(12) See, e.g., Landau, L. D.; Lifschitz, E. M. Elastizitātstheorie; Akademie-Verlag: Berlin, 1975.

(13) (a) Nelson, D. R.; Peliti, L. J. Phys. 1987, 48, 1085. (b) Seung, H. S.; Nelson, D. R. Phys. Rev. A, Gan. Phys. 1988, 38, 1005. (c) David, F.; Guitter, E.; Peliti, L. J. Phys. 1987, 48, 2059.

(14) See, e.g., Leibler, S.; Maggs, A. Phys, Rev. Lett. 1989, 63, 406.

(15) Lipowsky, R.; Girardet, M.' Phys. Rev. Lett. 1990, 65, 2893.

(16) See, e.g., Marra, J. J. Colloid Interface Sci. 1986, 109, 11.

(17) R. Lipowsky und S. Leibler, Phys. Rev. Lett. 1986, 56, 2541; 1987, $59,1983(\mathrm{E})$.
DGDG membranes. ${ }^{9}$ Very similar transitions also occur when a crumpled membrane is adsorbed onto a wall. ${ }^{18}$

A. Theoretical Models for the Conformations of Interacting Membranes. Now, consider two membrane segments with effective bending rigidities, $\kappa_{1} \approx K_{1} / q^{\eta}$ and $\kappa_{2} \approx K_{2} / q^{\eta}$, which are, on average, parallel. The effective Hamiltonian for their local separation, $l(x)$, or its Fourier transform, $l(q)$, is taken to be ${ }^{2}$

$\mathscr{H}\{l\}=\int \mathrm{d}^{2} q(\kappa / 2) q^{4-\eta}|\tau(q)|^{2}+\int \mathrm{d}^{2} x V[l(x)]$

with the reduced rigidity $K=K_{1} K_{2} /\left(K_{1}+K_{2}\right)$ and the membrane interaction

$$
\begin{aligned}
V(l) & =\infty & & \text { for } l \leq 0 \\
& =P l+V_{\mathrm{DI}}(l) & & \text { for } l>0
\end{aligned}
$$

The membranes are assumed to be in thermal equilibrium at temperature $T$; the statistical weight for a certain conformation, $l(x)$, is then given by the Boltzmann factor, $\exp [-\mathscr{H}\{l\} / T]$. The same model also applies to a membrane interacting with a solid surface or any other interface if one considers the limit $K_{2} \rightarrow \infty$.

The first term in the effective Hamiltonian (3.1) represents the $l$-dependent part of the bending energies. For fluid membranes, one has $\eta=0$, while $\eta>0$ for crystalline or polymerized membranes, as mentioned. The membrane interaction, $V(l)$, contains a hard wall at $l=0$ since the membranes cannot penetrate one another. For positive values of $l$, this interaction represents the free energy increase resulting (i) from an external pressure, $P$, and (ii) from the direct interaction, $V_{\mathrm{DI}}$, of the membranes, which reflects the nature of the underlying molecular forces. In general, this direct interaction includes (i) "nonspecific" interactions such as hydration, van der Waals, or electrostatic forces and (ii) "specific" interactions, which are mediated by biologically relevant macromolecules.

B. Renormalization Group Approach. As mentioned, the direct interaction is renormalized by thermally excited shape fluctuations of the membranes. This renormalization can be theoretically studied by a functional renormalization group (RG) approach which has been previously described in some detail. ${ }^{1}$ Thus, we will only give a brief summary here. First of all, the RG calculations predict that lipid bilayers interacting with realistic intermembrane forces can undergo such a transition at a critical unbinding temperature, $T=T_{\mathrm{u}}$. Alternatively, one may vary any parameter of the interaction such as, e.g., the Hamaker constant as discussed below. Then, the unbinding transition will occur at a critical value of this parameter.

In order to be more specific, let us consider lipid bilayers in water and assume that (i) the bilayers carry no electric charge and (ii) there are no macromolecules attached to them. Then, the direct interaction consists of two parts: (a) a strong short-ranged repulsion termed hydration interaction, which presumably arises from the local ordering of the water molecules close to the polar head groups of the lipids, and (b) a longer-ranged van der Waals attraction resulting from the polarizabilities of the water and lipid molecules. For two identical membranes, the van der Waals term is always attractive and favors a small value of the membrane separation $l$; the hydration term, on the other hand, is usually repulsive and favors a large value of $l$. The direct interaction is then given by ${ }^{19}$

(18) Lipowsky, R.; Baumgärtner, A. Phys. Rev. A: Gen. Phys, 1989, $40,2078$. 


$$
\begin{aligned}
& V_{\mathrm{DI}}(l)=B_{\mathrm{H}} \exp \left(-l / l_{\mathrm{H}}\right)-(W / 12 \pi)\left[1 /\left(l^{2}+l_{0}{ }^{2}\right)-\right. \\
&\left.2 /\left(l+l_{\mathrm{M}}\right)^{2}+1 /\left(l+2 l_{\mathrm{M}}\right)^{2}\right]
\end{aligned}
$$

Typical values for the empirical hydration interaction are $B_{\mathrm{H}} \simeq 0.2 \mathrm{~J} / \mathrm{m}^{2}$ and $l_{\mathrm{H}} \simeq 0.3 \mathrm{~nm}$. The Hamaker constant, $W$, of the van der Waals interaction is positive for two identical membranes but could be positive or negative for a membrane interacting with another interface; this constant is typically of order $10^{-22}-10^{-21} \mathrm{~J}$. The length scale $l_{0}$ is a microscopic cutoff which ensures that there is no divergence at small $l$, and $l_{\mathrm{M}} \simeq 4 \mathrm{~nm}$ denotes the membrane thickness.

Functional renormalization of this interaction leads to critical values, $W_{\mathrm{u}}$, for the Hamaker constant that are well within the experimentally accessible range. For example, two fluid membranes at room temperature $T=T_{0}=4.114$ $\times 10^{-21} \mathrm{~J}$ with bending rigidities $\kappa_{1}=\kappa_{2}=2 \kappa \simeq 10 T_{0}$ are predicted to unbind at the critical value $W_{\mathrm{u}} \simeq T_{0}$ of the Hamaker constant. 17

Another important prediction of the RG work is that these transitions should be continuous and characterized by universal critical behavior. ${ }^{2,17,20}$ For example, the mean separation, $\langle l\rangle$, of the membranes grows as

$$
\langle l\rangle \sim 1 /\left(T_{\mathrm{u}}-T\right)^{\psi} \sim 1 /\left(W-W_{\mathrm{u}}\right)^{\psi}
$$

as the unbinding temperature, $T_{\mathrm{u}}$, or the critical Hamaker constant, $W_{\mathrm{u}}$, are approached. The critical exponent $\psi$ is independent of the parameters of the direct interaction; it only depends on the internal structure of the membranes: the RG approach leads to the presumably exact value $\psi=1$ for fluid membranes ${ }^{2}$ and to the estimate $\psi$ $\simeq 0.7<1$ for polymerized membranes. ${ }^{2,21}$

For $T \geq T_{\mathrm{u}}$, the unbound membranes are scaleinvariant: on average, their conformation does not change under the rescaling transformation ${ }^{1}$

$$
x \rightarrow x / b \text { and } l \rightarrow l / b^{\zeta}
$$

with arbitrary rescaling factor $b>1$ and roughness exponent $\zeta=(2-\eta) / 2$. For $T<T_{\mathrm{u}}$, this scale-invariance is broken on $x$ scales of the order of the longitudinal correlation length, $\xi_{\|}$, which satisfies the relation $\langle l\rangle \sim$ $\xi_{\|} \zeta .22$

C. Monte Carlo Studies of Fluid Membranes. Renormalization group methods are expected to give reliable values for the critical exponents of the unbinding transitions. On the other hand, it is difficult to assess the accuracy of this approach for the unbinding temperature, $T_{\mathrm{u}}$, or for the critical Hamaker constant, $W_{\mathrm{u}}$. In order to get more reliable estimates for these latter quantities, Monte Carlo (MC) studies of the effective Hamiltonian (3.1) have been performed for fluid membranes (i.e., for $\eta$ $=0){ }^{3}$ These numerical studies show that the RG predictions give the right order of magnitude for $T_{\mathrm{u}}$ or $W_{\mathrm{u}}$. In addition, the simulations reveal a rather strong dependence of $T_{\mathrm{u}}$ on the small-distance cutoff, $a$, and thus on the molecular structure of the membrane.

In the MC work, the spatial coordinate, $x$, is replaced by a square lattice with lattice sites, $\left\{x_{i}\right\}$, the lattice constant,

(19) See, e.g., Parsegian, V. A.; Fuller, N.; Rand, R. P. Proc. Natl. Acad. Sci. USA 1979, 76, 2750.

(20) Grotehans, S.; Lipowsky, R. Phys. Rev. A: Gen. Phys. 1990, 41 4574.

(21) This value has been obtained in ref 2 from functional renormalization with rescaling factor $b=2$.

(22) If fluid membranes have a finite persistence length, $\xi$ even in the presence of self-avoidance, they will exhibit a different scaling behavio for $x \gg \xi_{p}$. For example, if they resemble branched polymers on large scales, the radius of gyration for a segment of linear size $L_{\text {in }}$ would scale as $L_{\text {in }}$ " with $\nu \simeq 1$ in three dimensions.

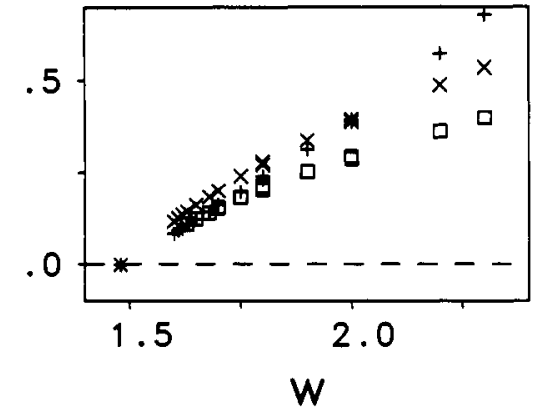

Figure 1. Unbinding transition for the square well interaction as defined by (3.7). The symbols $\square, \times$, and + represent MC data for the inverse length scales $1 / \hat{\xi}_{1}, 1 / \hat{\xi}_{\|}$, and $1 / l$, respectively.

$a$. The membrane configuration is then specified by $l_{i} \equiv$ $l\left(x_{i}\right)$, and the effective Hamiltonian becomes

$\mathscr{H}\left\{l_{i}\right\} / T=\sum_{i}\left\{{ }^{1} /{ }_{2}\left(\kappa / T a^{2}\right)\left(\nabla_{\mathrm{d}}{ }^{2} l_{i}\right)^{2}+a^{2} V\left(l_{i}\right) / T\right\}$

with the discrete Laplacian $\nabla_{\mathrm{d}}{ }^{2} l_{i} \equiv l\left(x_{i}+a \hat{x}_{1}\right)+l\left(x_{i}-a \hat{x}_{1}\right)$ $+l\left(x_{i}+a \hat{x}_{2}\right)+l\left(x_{i}-a \hat{x}_{2}\right)-4 l\left(\hat{x}_{i}\right)$. It is convenient to use the dimensionless variables $z_{i} \equiv(\kappa / T)^{1 / 2} l_{i} / a$ and $U\left(z_{i}\right) \equiv$ $a^{2} V\left[(T / \kappa)^{1 / 2} a z_{i}\right] / T$ in order to reduce the number of parameters in the effective Hamiltonian.

First, consider the square well interaction defined by

$$
\begin{aligned}
V(l) & =\infty & & \text { for } l<0 \\
& =-W & & \text { for } 0<l<l_{0} \\
& =0 & & \text { for } l_{0}<l
\end{aligned}
$$

which has been studied in great detail. ${ }^{3}$ In this case, the rescaled potential, $U(z)$, depends only on the two parameters $z_{0} \equiv(\kappa / T)^{1 / 2} l_{0} / a$ and $w \equiv a^{2} W / T$. For each value of $z_{0}$, one may locate a critical value, $w_{\mathrm{u}}=w_{\mathrm{u}}\left(\boldsymbol{z}_{0}\right)$, of the rescaled potential strength. ${ }^{3}$ In practice, a good estimate of $w_{\mathrm{u}}$ can be obtained from the $w$-dependence of the dimensionless length scales

$$
l \equiv\left[\langle l\rangle-l_{0} / 2\right] / l_{\mathrm{H}}, \hat{\xi}_{\perp} \equiv\left\langle[l-\langle l\rangle]^{2}\right\rangle^{1 / 2} / l_{\mathrm{H}}
$$

and

$$
\hat{\xi}_{\|} \equiv \exp \left[2 \pi(\kappa / T)\left\langle(\nabla l)^{2}\right\rangle\right]
$$

Note that $\hat{\xi}_{\|}$is a length scale measured in units of the lattice constant, $a$, while the length scales $l$ and $\hat{\xi}_{\perp}$ are measured in units of the hydration length, which is taken to be $l_{\mathrm{H}}=0.3 \mathrm{~nm}$. The $w$-dependence of the inverse quantities $1 / l, 1 / \hat{\xi}_{\perp}$, and $1 / \hat{\xi}_{\|}$is shown in Figure 1 for $z_{0}$ $=0.075$.

The realistic interaction as given by (3.3), which represents the superposition of hydration and van der Waals interaction, has also been studied by MC simulations for some values of the interaction parameters. ${ }^{23}$ In this case, the rescaled potential, $U(z)$, depends on five dimensionless parameters. In order to explore a part of the phase diagram that is experimentally accessible, the following parameter values have been chosen: $B_{\mathrm{H}}=0.2 \mathrm{~J} / \mathrm{m}^{2}, l_{\mathrm{H}}=$ $0.3 \mathrm{~nm}, l_{0}=l_{\mathrm{H}}, l_{\mathrm{M}}=4 \mathrm{~nm}, a=l_{\mathrm{M}}$, and $T=T_{0}=4.114 \times$ $10^{-21} \mathrm{~J}$. Then, the critical value, $W_{\mathrm{u}}$, has been estimated for several values of $\kappa$.

An example is shown in Figure 2 for $\kappa_{1}=2 \kappa=2 T_{0}$. In this figure, the inverse length scales $1 / \hat{\xi}_{\perp}$ and $1 / \hat{\xi}_{\|}$have been plotted as a function of the dimensionless parameter $w \boxminus W_{\kappa} / 12 \pi T^{2}$ with $T=T_{0}$ and $\kappa=2 T_{0}$. In this case, the critical value is estimated to be $w_{\mathrm{u}}=0.088 \pm 0.007$ or $W_{\mathrm{u}}$ $=(13.6 \pm 1.0) \times 10^{-21} \mathrm{~J}$. In the same way, the critical

(23) Lipowsky, R.; Zielinska, B., unpublished. 


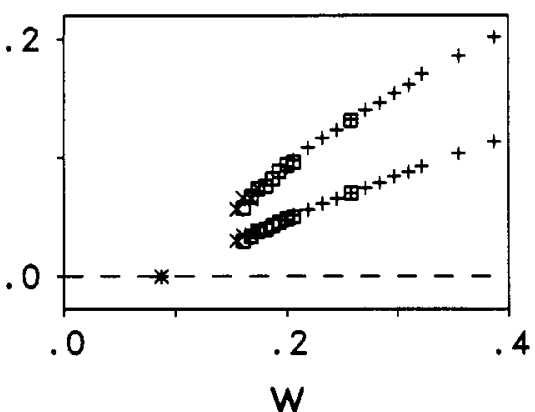

Figure 2. Unbinding transition for the realistic interaction as given by (3.3). The upper and the lower curve represent MC data for $1 / \hat{\xi}_{\perp}$ and $1 / 2 \xi_{\|}$, respectively.

values $W_{\mathrm{u}}=(3.0 \pm 0.5) \times 10^{-21} \mathrm{~J}$ and $W_{\mathrm{u}}=(1.5 \pm 1.0) \times$ $10^{-21} \mathrm{~J}$ have been found for $\kappa_{1}=0.2 \sim 10^{-19} \mathrm{~J}$ and $\kappa_{1}=0.4$ $\times 10^{-19} \mathrm{~J}$, respectively. ${ }^{23}$ These values apply to the choice $a=l_{\mathrm{M}}=4 \mathrm{~nm}$ for the small-distance cutoff. As mentioned, a change in this cutoff has a rather strong effect on these critical values: the data on the square well potential (3.7) indicate that $T_{\mathrm{u}}$ or $W_{\mathrm{u}}$ vary linearly with $a .^{3}$ Therefore, the phase boundaries for the unbinding transition will be strongly affected by defects, impurities, or other changes in the molecular structure of the membranes. Likewise, the microroughness proposed by Helfrich ${ }^{24}$ would have a strong effect on $T_{\mathrm{u}}$.

D. Relevance of Lateral Tension. So far, it has been assumed that the membranes experience no lateral tension, $\Sigma$. Now, assume that the two membranes feel a tension, $\Sigma_{1}$ and $\Sigma_{2}$, respectively. Then, the effective Hamiltonian becomes

$$
\mathscr{H}_{\Sigma}\{l\}=\mathscr{H}\{l\}+\int \mathrm{d}^{2} q^{1} /{ }_{2} \Sigma q^{2}|\tilde{l}(q)|^{2}
$$

with $\Sigma=\Sigma_{1} \Sigma_{2} /\left(\Sigma_{1}+\Sigma_{2}\right)$. It is now assumed that, for $\Sigma$ $=0$, the effective Hamiltonian $\mathscr{H}\{l\}$ contains a direct interaction as in (3.3) which leads to an unbinding transition at $T=T_{\mathrm{u}}$. Thus, for $\Sigma=0$, the membranes are bound and unbound for $T<T_{\mathrm{u}}$ and for $T>T_{\mathrm{u}}$, respectively. Then, a finite lateral tension, $\Sigma>0$, will lead to a bound state at any $T<\infty$ and thus will suppress the unbinding transition. Indeed, the effective Hamiltonian $\mathscr{H}_{\Sigma}\{l\}$ with a direct interaction as in (3.3) will always lead to a bound state unless the Hamaker constant $W$ is decreased to zero. ${ }^{25}$

On the other hand, one may now ask what happens in the limit $\Sigma \rightarrow 0$. Then, the membranes attain a finite separation for $T<T_{\mathrm{u}}$ but continuously unbind for $T \geq$ $T_{\mathrm{u}}$. Rescaling the $\Sigma$ term in the effective Hamiltonian (3.10) according to (3.5), one obtains the transformation

$$
\Sigma \rightarrow \Sigma^{\prime}=b^{2 \zeta} \Sigma \text { for } T \geq T_{\mathrm{u}}
$$

Then, standard scaling arguments imply that the longitudinal correlation length, $\xi_{\|}$, and the mean separation, $l$, behave as

$$
\xi_{\|} \sim \Sigma^{-1 / 2 \zeta} \text { and }\langle l\rangle \sim \xi_{\|}^{\zeta} \sim \Sigma^{-1 / 2}
$$

for $T \geq T_{\mathrm{u}}$ as $\Sigma$ goes to zero. Likewise, the adhesion free energy, $f_{\mathrm{a}}$, has a singular part which scales as $f_{\mathrm{a}} \sim-T / \xi_{\|}{ }^{2}$ $\sim-\Sigma^{1 / \zeta}$. For fluid membranes, the scaling behavior for $l$ and $f_{\mathrm{a}}$ has been previously obtained by different arguments. ${ }^{26}$

(24) Helfrich, W. Lig. Cryst., in press.

(25) This follows from results for wetting transitions, see Lipowsky, R. Phys. Reu. Lett. 1984, 52, 1429.

(26) Helfrich, W.; Servuss, R. M. Nuovo Cimento D 1984, 3, 137.
E. Irrelevance of Quenched Disorder. Another type of perturbation that could affect the unbinding behavior is the presence of frozen or quenched disorder. For example, the direct interaction between a fluid or polymerized membrane and a solid surface may contain a random part resulting from defects or impurities at the surface. Then the strength, $W$, of the attractive part of the direct interaction, $V_{\mathrm{DI}}$, will be a (quenched) random variable, which may be characterized by the expectation value $\langle W\rangle$ $\boxminus W_{0}$, and its variance $\left\langle[W-\langle W\rangle]^{2}\right\rangle \equiv(\Delta W)^{2}$.

Close to an unbinding transition, the membrane consists of correlated segments that have a linear extension $\sim \xi_{\|}$. Then, alluding to the central limit theorem, the total adhesion energy for such a segment has a mean value $\sim W_{0} \xi_{\|}{ }^{2}$ and a standard deviation $\sim \Delta W \xi_{\|}$. Therefore, the unbinding temperature $T_{\mathrm{u}}(W)$ of a $\xi_{\|}$segment is of order $T_{\mathrm{u}}\left(W_{0}\right) \pm \Delta W / \xi_{\|}$and thus exhibits fluctuations of order $\Delta T_{\mathrm{u}} \simeq \Delta W / \xi_{\|}$.

Now, one must ask if these fluctuations in $T_{\mathrm{u}}$ change the critical behavior at the transition. Thus, let us first assume that these fluctuations are irrelevant. The unbinding transition will then occur at $T_{\mathrm{u}}=T_{\mathrm{u}}\left(W_{0}\right)$ with the same critical exponents as in the absence of disorder. Such an assumption is only consistent if the fluctuations, $\Delta T_{\mathrm{u}}$, of the unbinding temperature are small compared to the temperature deviation, i.e., if $\Delta T_{\mathrm{u}} \ll T_{\mathrm{u}}\left(W_{0}\right)-T$ as the transition temperature is approached from below. In such a situation, the correlation length $\xi_{\|}$would diverge as $\xi_{\|}$ $\sim\left[T_{\mathrm{u}}\left(W_{0}\right)-T\right]^{-v_{\|}}$, and the fluctuations in the unbinding temperature would vanish as $\Delta T_{\mathrm{u}} \sim\left[T_{\mathrm{u}}\left(W_{0}\right)-T\right] \nu^{\nu_{1}}$. Thus, the assumption of irrelevant disorder is only self-consistent provided

$$
\begin{array}{r}
\Delta T_{\mathrm{u}} \sim 1 / \xi_{\|} \sim\left[T_{\mathrm{u}}\left(W_{0}\right)-T\right]^{\nu_{1}} \ll T_{\mathrm{u}}\left(W_{0}\right)-T \\
\quad \text { or } \nu_{\|}>1
\end{array}
$$

For fluid membranes, one has $\nu_{\|}=1$ and thus a marginal case according to (3.13). Then, the critical behavior will presumably exhibit confluent logarithmic singularities while the critical exponents are expected to remain unchanged. For polymerized membranes, the RG calculations lead to the estimate $\nu_{\|} \simeq 1.3>1.2,21$ The criterion (3.13) then leads to the conclusion that, to leading order, quenched disorder does not affect the critical behavior at continuous unbinding transitions. ${ }^{27}$

\section{Adhesion of Vesicles}

In the previous section, we considered the behavior of a membrane segment bound to another surface (which could be a second membrane). So far, we did not worry about the edges of such a segment. In aqueous solution, the edge of a lipid bilayer has a finite edge tension (or edge free energy) per unit length arising from the contact between the water and the hydrocarbon chains. Therefore, a sufficiently large membrane segment in solution will form a closed surface or vesicle in order to avoid this tension. ${ }^{28}$

The average shape of vesicles has been studied in many experiments, primarily by light microscopy. ${ }^{5}$ In addition to the average shape, these experiments also give direct evidence for thermally excited shape fluctuations. ${ }^{29}$ Vesicles frequently interact with other membranes or inter-

(27) For direct interactions that exhibit several minima, the surfaces can undergo multicritical unbinding transitions at which quenched disorder should change the critical behavior; see ref 20 .

(28) For a bound membrane segment, the total adhesion energy scales like the area of the membrane and thus will always overcome the edge tension for sufficiently large segments.

(29) For a review, see Sackmann, E.; Duwe, H. P.; Engelhardt, H. Faraday Discuss. Chem. Soc. 1986, 81, 281. 
faces. If this interaction is attractive, it can lead to a bound state of the vesicle. Such bound states can be experimentally investigated by micropipet techniques. ${ }^{30}$ In this latter case, one studies the adhesion of two vesicles, one of which is sucked into a micropipet.

From a theoretical point of view, the shape of a bound vesicle is determined by the interplay of adhesion and bending energies. This interplay can be systematically studied within a simple model as will be described below. ${ }^{4}$ Within this model, we obtain a large variety of different shapes. A few examples are shown in Figure 3 and Figure 5 below. In addition, we find nontrivial adhesion transitions between free and bound vesicles as well as transitions between two different bound states, which occur, e.g., when the temperature or the osmotic conditions are changed. Finally, our theory helps to clarify the notion of a contact angle for membranes.

A. Bound States of Vesicles. Now, consider a membrane that forms a closed surface with total surface area, $A$, and enclosed volume, $V$. The membrane is attracted to a wall by a contact potential, $W$, which represents an adhesion energy per unit area. The free energy functional of such a vesicle is then given by ${ }^{4}$

$$
\begin{aligned}
F & =F_{\kappa}+F_{\mathrm{W}}+F_{\mathrm{P}}+F_{\Sigma} \\
& =(\kappa / 2) \oint \mathrm{d} A\left(C_{1}+C_{2}-C_{0}\right)^{2}-W A^{*}+P V+\Sigma A
\end{aligned}
$$

The first term represents the surface integral over the bending energy of the membrane where $C_{1}$ and $C_{2}$ are the two principal curvatures. ${ }^{6}$ The spontaneous curvature $C_{0}$ is a phenomenological parameter that reflects a possible asymmetry of the inner and outer monolayer. For charged membranes, $C_{0}$ may be changed by adding salt to the interior or exterior water medium. We do not include any energy related to the stretching of the membrane. Thus, we assume that the membrane is fluid. In addition, we also assume that the vesicle does not change its topology and, thus, does not include the Gaussian curvature.

The second term, $-W A^{*}$, in (4.1) represents the adhesion free energy. We are mainly interested in the global shape of a bound vesicle with a typical size of the order of micrometers and, thus, neglect spatial variations on the scale of nanometers, which is the typical range of interaction potentials. Thus we have replaced this microscopic potential by a contact potential of strength $W$. The gain in energy for a bound vesicle state is then proportional to the contact area, $A^{*}$.

The last two terms in (4.1) refer to the external constraints imposed on the total surface area, $A$, and on the total volume, $V$, of the vesicle. In some experiments, the osmotic conditions are not changed while the area of the vesicle is increased, e.g., by raising the temperature. This defines the $(V, A)$ ensemble for which $P$ and $\Sigma$ denote Lagrange multipliers. On the other hand, if the pressure is experimentally controlled, e.g., by the osmotic conditions, one has to include an additional free energy change $P \mathrm{~d} V$, where $P$ denotes the difference between the exterior and interior pressure: $P \equiv P_{\text {ext }}-P_{\text {int }}$. This latter situation defines the $(P, A)$ ensemble.

For $W=0$, i.e., without the adhesion term, the theoretical model as defined by (4.1) has been studied in some detail. ${ }^{6,31-34}$ If one assumes that the shape is axisymmetric, this shape can be uniquely parameterized by the angle,

(30) For a review, see Evans, E. Colloids Surf. 1990, 43, 327.

(31) Deuling, H. J.; Helfrich, W. J. Phys. 1976, 37, 1335.

(32) Jenkins, J. T. J. Math. Biol. 1977, 4, 149.

(33) Peterson, M. A. J. Appl. Phys. 1988, 57, 1739.

(34) Seifert, U.; Berndl, K.; Lipowsky, R. Phys. Rev. A, in press.

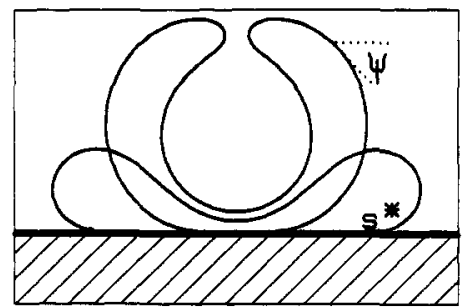

Figure 3. Contours for two axisymmetric shapes which are bound to another surface. The contour is parametrized by the angle $\Psi$ as a function of the arclength $s$. The contact point is at $s=s^{*}$.

$\Psi=\Psi(s)$, which determines the orientation of the surface normal as a function of the arclength, $s$, along the contour; comapre Figure 3 . One then has to solve a set of ordinary but nonlinear differential shape equations, which are obtained by minimization of the free energy (4.1). This leads to several branches of one-parameter families of solutions. Branches of lowest energy are (i) prolate ellipsoids, (ii) oblate ellipsoids that transform smoothly to discocyte-type shapes if the pressure is increased or the volume decreased, and (iii) stomatocyte-type shapes with broken up/down symmetry. ${ }^{34}$

For bound vesicles, these shape equations remain valid since the additional term $F_{\mathrm{W}}$ enters only as a boundary condition. Two such conditions have to be imposed along the line of contact: (i) since any sharp bent costs an infinite amount of bending energy (in the absence of defects), the membrane must touch the wall tangentially, i.e., the contact angle $\Psi$ ( $s=s^{*}$ ) is identical with $\pi$; (ii) in addition, minimization of (4.1) with a variable contact line leads to a nontrivial boundary condition for the contact curvature which is given by ${ }^{4}$

$$
1 / R_{1}{ }^{*} \equiv C_{1}{ }^{*}=\mathrm{d} \Psi(s) /\left.\mathrm{d} s\right|_{s^{*}}=(2 W / k)^{1 / 2}
$$

Thus, the contact potential $W$ and the bending ridigity $\kappa$ completely determine the curvature at $s$ and $s^{*}$. This relation is independent of the size of the vesicle, of the spontaneous curvature, and of the pressure. It holds in any ensemble.

We have performed a systematic study of the shape equations with these boundary conditions. ${ }^{4}$ As a result, we found a large variety of different shapes. A few examples are shown in Figure 3 and Figure 5.

For bound vesicle shapes which minimize the energy as given by (4.1), the boundary condition (4.2) holds as a strict equality. A similar but approximate relation given by $\max \left(C_{1}\right) \simeq(2 \mathrm{~W} / \kappa)^{1 / 2}$ has been found for the maximum curvature, $\max \left(C_{1}\right)$, at the edge of the contact when the membrane is bound by a long-ranged attractive potential of strength $W .^{30}$

B. Adhesion Transition. From a theoretical point of view, the most relevant vesicle states are those of lowest energy. For a given set of parameters, the state of lowest energy that may be bound or free defines a phase diagram. This phase diagram depends on the ensemble: in the $(P, A)$ ensemble, one has to determine the minimum of $F_{k}+F_{\mathrm{W}}$ $+F_{\mathrm{P}}$, while in the $(V, A)$ ensemble only $F_{\mathrm{x}}+F_{\mathrm{W}}$ are minimized. The $(P, A)$ ensemble has been discussed elsewhere. ${ }^{4}$ Here, we will focus on the $(V, A)$ ensemble with zero spontaneous curvature, i.e., with $C_{0}=0$.

Because of the scale invariance of the free energy (4.1), it is convenient to introduce the dimensionless quantities

$$
w \equiv W R^{2} / \kappa \text { and } v \equiv V /\left((4 / 3) \pi R^{3}\right)
$$

with $R \equiv(A / 4 \pi)^{1 / 2}$. The corresponding phase diagram is shown in Figure 4. This phase diagram is basically divided into two parts by the lines $\left(\mathrm{D}_{\mathrm{a}}^{\mathrm{pr}}, \mathrm{D}_{\mathrm{a}}^{\mathrm{ob}}, \mathrm{D}_{\mathrm{a}}^{\mathrm{st}}, \mathrm{C}_{\mathrm{a}}^{\mathrm{st}}\right)$, which 


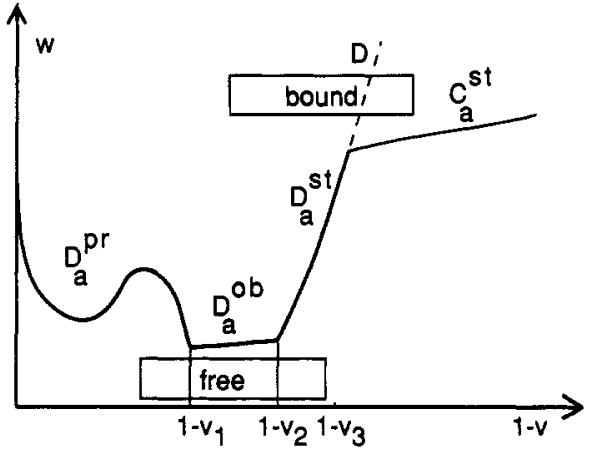

Figure 4. Phase diagram for vesicles with enclosed volume, $v$, which are attracted by a contact potential of strength, $w$. The vesicle is free for small $w$ and bound for large $w$. All vesicles have the same surface area.

define a critical adhesion potential $w_{\mathrm{a}}(v)$. For $w>w_{\mathrm{a}}(v)$, the state of lowest energy is bound, while for $w<w_{\mathrm{a}}(v)$ it is free even in the presence of an attractive wall. This free state is a prolate ellipsoid for $1>v \geq v_{1} \simeq 0.65$, a discocytetype shape (which branches off from the oblate ellipsoid) for $v_{1} \geq v \geq v_{2} \simeq 0.59$, and a stomatocyte-type shape for $v_{2}>v{ }^{34}$ The lines $\left(\mathrm{D}_{\mathrm{a}}^{\mathrm{pr}}, \mathrm{D}_{\mathrm{a}}{ }^{\mathrm{ob}}, \mathrm{D}_{\mathrm{a}}^{\mathrm{st}}\right)$ denote discontinuous adhesion transitions between these free states and a bound state with finite contact area $A^{*}$. For $v<v_{3} \simeq 0.52$, two different bound states coexist along the line (D). In fact, the two shapes displayed in Figure 3 represent an example for this coexistence. The contact area of the bound stomatocyte-type state vanishes continuously for $w \rightarrow w_{\mathrm{a}}(v)$ from above, i.e. the line $\left(\mathrm{C}_{\mathrm{a}}{ }^{\mathrm{at}}\right)$ denotes a line of continuous adhesion transitions.

Thus, the phase diagram for the $(V, A)$ ensemble has the same basic features as the phase diagram for the $(P, A)$ ensemble, which has been displayed in ref 4: A free vesicle state for weak contact potential undergoes an adhesion transition to a bound vesicle state as the strength of the contact potential is increased. This transition is governed by the competition between bending and adhesion energies.

The bending energy is scale invariant, i.e. independent of the size of the vesicle, while the gain in adhesion energy is proportional to the area of the vesicle. Therefore, the critical value of the contact potential, $W=W_{\mathrm{a}}=w_{\mathrm{a}} \kappa / R^{2}$, is also size-dependent. Thus, for fixed $W$, vesicles of linear size $R>R_{\mathrm{a}} \equiv\left(w_{\mathrm{a}} \kappa / W\right)^{1 / 2}$ are bound while those of linear size $R>R_{\mathrm{a}}$ are free. This prediction is based on minimization of the free energy functional defined by (4.1). In real systems, at least two additional effects will influence this transition: (i) since the vesicle has a finite size, thermally activated unbinding of vesicles preempts the adhesion transition, if the energy difference between bound and free states becomes of the order of $\mathrm{T}$; (ii) shape fluctuations of the adjacent part of the membrane may also lead to an unbinding of vesicles via the mechanism described in section III. A rough estimate of the fluctuation effects leads to a crossover radius $R_{\mathrm{c}} \simeq 0.2 \mu \mathrm{m}:{ }^{4}$ large vesicles with $R \gg R_{\mathrm{c}}$ unbind via thermally excited shape fluctuations while smaller vesicles with $R \ll R_{\mathrm{c}}$ undergo an adhesion transition as discussed in this section.

C. Notion of an Effective Contact Angle. Within the continuum model used here, the contact angle for vesicle adhesion is identical with $\pi$ as discussed in section IV.A and, thus, is not a particularly interesting quantity. In fact, it is the contact curvature which is determined by the boundary condition (4.2) in a nontrivial way. On the other hand, let us now consider the related problem of adhesion of a liquid droplet on a solid substrate. In the

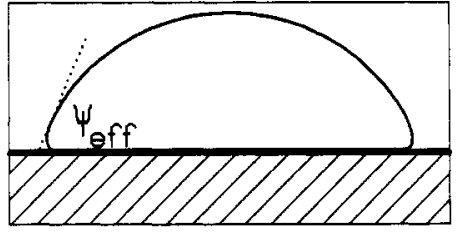

Figure 5. Shape of a bound vesicle with effective contact angle $\Psi_{\text {eff. }}$ Such an angle can be defined provided (i) the interior pressure exceeds the exterior pressure, and (ii) the radius of curvature, $\xi \mathrm{w}$, at the contact point is much smaller than the linear size of the vesicle.

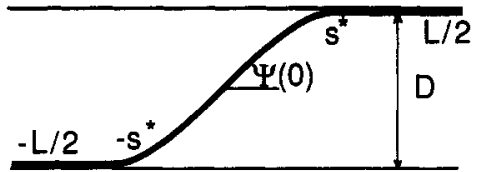

Figure 6. Adhesion of a membrane to two parallel surfaces at separation $D$. The shape is parametrized by the angle $\Psi=\Psi(s)$ with $-L / 2 \leq s \leq L / 2$. The contact points are at $s= \pm s^{*}$.

latter situation, the contact angle depends on the various interfacial tensions and is given by the Young-Dupre equation. The basic difference between both phenomema is the bending elasticity, which is usually unimportant for the adhesion of liquid droplets.

If the bending rigidity $\kappa$ becomes small, sharp bents cost only a small energy and one might expect to recover the Young-Dupre equation even for vesicle adhesion. This is indeed the case in the following sense: For small $\kappa$ (or equivalently for large $W$ ), the contact curvature $C_{1}{ }^{*}=$ $(2 W / \kappa)^{1 / 2}$ becomes large, i.e., the radius of curvature, $R_{1} *$ $=(\kappa / 2 \mathrm{~W})^{1 / 2}$, at the contact point becomes small. If this length scale is much smaller than the linear size, $R$, of the vesicle, the shape approximates a spherical cap as shown in Figure 5. In the $(P, A)$ ensemble, this corresponds to $P$ $=P_{\text {ext }}-P_{\text {int }}<0$, i.e., to an interior pressure that exceeds the exterior pressure. For such a shape, one may define an effective contact angle, $\Psi_{\text {eff }}$, which indeed obeys the Young-Dupre equation

$$
W=\Sigma\left(1+\cos \Psi_{\text {eff }}\right)
$$

For fixed area $A$, the lateral tension $\Sigma$ has the numerical value of the Lagrange multiplier required for this area. On the other hand, this tension can also be related to the negative pressure $P$ and to the radius $R_{\text {eff }}$ of the spherical cap via the Laplace equation $P=-2 \Sigma / R_{\text {eff. }}$.

Finally, let us briefly discuss the adhesion geometry shown in Figure 6 which is motivated by the conformations of membranes in multilayer systems such as, e.g., in large liposomes. The membrane is attracted to two different "walls" at separation, $D$. It feels a contact potential, $W$, and, in addition, a lateral tension, $\Sigma$, acting parallel to the walls. For simplicity, the membrane is taken to bend only along one spatial direction so that its shape can be parametrized by the angle $\Psi=\Psi(s)$ with $-L / 2<s<L / 2$ as indicated in Figure 6 . The free energy, $f$, per unit length is then given by

$$
\begin{array}{r}
f=\int_{-L / 2}^{L / 2} \mathrm{~d} s\left\{(\kappa / 2)(\mathrm{d} \Psi / \mathrm{d} s)^{2}+\Sigma(1-\cos \Psi)-\lambda \sin \Psi\right\}- \\
W\left(L-2 s^{*}\right)
\end{array}
$$

where $\lambda$ is a Lagrange multiplier in order to ensure that $\int \mathrm{d} s \sin \Psi=D$. The boundary conditions at the contact line are again given by $\Psi\left( \pm s^{*}\right)=0$ and $\mathrm{d} \Psi /\left.\mathrm{d} s\right|_{ \pm g^{*}}=$ $\mp(2 \mathrm{~W} / \kappa)^{1 / 2}=\mp 1 / R_{1} *$.

Minimization of the free energy (4.5) leads to a shape equation which can be solved in terms of elliptic functions. One then finds that, for $R_{1} * \ll D$, the angle $\Psi(\mathrm{s})$ is roughly 
constant and given by $\Psi(s) \simeq \Psi(0)$ for the intermediate $s$ range $-\left(s^{*}-R_{1}^{*}\right) \lessgtr s \lesssim s^{*}-R_{1}^{*}$. The slope $\Psi(0)$ then satisfies the Young-Dupre equation $W=\Sigma_{0}[1-\cos \Psi(0)]$, where $\Sigma_{0} \equiv \Sigma / \cos \Psi(0)$ can be regarded as the tension acting in the middle of the membrane. ${ }^{35}$ Therefore, an effective contact angle $\Psi_{\text {eff }}=\Psi(0)$ can be defined for $R_{1}$ * $\ll D$. On the other hand, for $R_{1}{ }^{*} \gg D$, the slope $\Psi(s)$ of the membrane varies over the whole range $-s^{*}<s<s^{*}$, and it makes no sense to talk about an effective contact angle. A similar distinction applies to the adhesion geometry discussed by Servuss and Helfrich. ${ }^{36}$

In summary, the notion of an effective contact angle for vesicle and membrane adhesion is meaningful only if the

(35) Compared to (4.2), there is an additional minus sign which arises from $\Psi \rightarrow \pi-\Psi$. typical length scale of the adhesion geometry is large compared to the scale $R_{1}{ }^{*}$, which represents the radius of curvature at the contact line. This effective contact angle obeys an appropriate Young-Dupre equation, which is then a consequence of the universal boundary condition (4.2) for the contact curvature.

Acknowledgment. We thank Wolfgang Helfrich and Erich Sackmann for helpful discussions and acknowledge support by the Sonderforschungsbereich 266 der Deutschen Forschungsgemeinschaft and by the Höchstleistungsrechenzentrum of the KFA Jülich.

(36) Servuss, R. M.; Helfrich, W. J. Phys. Fr. 1989, 50, 809. 